\title{
A molecular imprinting-based turn-on Ratiometric fluorescence sensor for highly selective and sensitive detection of 2,4-dichlorophenoxyacetic acid (2,4-D)
}

\author{
Xiaoyan Wang ${ }^{\mathrm{a}, \mathrm{b}, \mathrm{c}}$, Jialuo $\mathrm{Yu}^{\mathrm{a}, \mathrm{b}}$, Xiaqing Wu ${ }^{\mathrm{b}}$, Junqing Fu ${ }^{\mathrm{b}}$, Qi Kang ${ }^{\mathrm{a}}$, Dazhong Shen ${ }^{\mathrm{a}, *}$, \\ Jinhua Li $^{\mathrm{b}}$, Lingxin Chen ${ }^{\mathrm{b}, *}$ \\ ${ }^{a}$ College of Chemistry, Chemical Engineering and Materials Science, Collaborative Innovation Center of Functionalized Probes for Chemical Imaging in \\ Universities of Shandong, Key Laboratory of Molecular and Nano Probes, Ministry of Education, Shandong Provincial Key Laboratory of Clean Production of \\ Fine Chemicals, Shandong Normal University, Jinan 250014, China \\ ${ }^{\mathrm{b}}$ Key Laboratory of Coastal Environmental Processes and Ecological Remediation, Yantai Institute of Coastal Zone Research, Chinese Academy of Sciences, \\ Yantai 264003, China \\ c School of Pharmacy, Binzhou Medical University, Yantai 264003, China
}

\section{A R T I C L E I N F O}

\section{Article history:}

Received 24 December 2015

Received in revised form

26 February 2016

Accepted 14 March 2016

Available online 15 March 2016

Keywords:

Molecular imprinting

Fluorescence turn-on

Photoinduced electron transfer

Ratiometric fluorescence

2,4-Dichlorophenoxyacetic acid (2,4-D)

Water sample

\begin{abstract}
A B S T R A C T
A novel molecular imprinting-based turn-on ratiometric fluorescence sensor was constructed via a facile sol-gel polymerization for detection of 2,4-dichlorophenoxyacetic acid (2,4-D) on the basis of photoinduced electron transfer (PET) by using nitrobenzoxadiazole (NBD) as detection signal source and quantum dots (QDs) as reference signal source. With the presence and increase of 2,4-D, the amine groups on the surface of $\mathrm{QDs} @ \mathrm{SiO}_{2}$ could bind with 2,4-D and thereby the NBD fluorescence intensities could be significantly enhanced since the PET process was inhibited, while the QDs maintained constant intensities. Accordingly, the ratio of the dual-emission intensities of green NBD and red QDs could be utilized for turn-on fluorescent detection of 2,4-D, along with continuous color changes from orange-red to green readily observed by the naked eye. The as-prepared fluorescence sensor obtained high sensitivity with a low detection limit of $0.14 \mu \mathrm{M}$ within $5 \mathrm{~min}$, and distinguished recognition selectivity for 2,4-D over its analogs. Moreover, the sensor was successfully applied to determine 2,4-D in real water samples, and high recoveries at three spiking levels of 2,4-D ranged from $95.0 \%$ to $110.1 \%$ with precisions below 4.5\%. The simple, rapid and reliable visual sensing strategy would not only provide potential applications for high selective ultratrace analysis of complicated matrices, but also greatly enrich the research connotations of molecularly imprinted sensors.
\end{abstract}

(c) 2016 Elsevier B.V. All rights reserved.

\section{Introduction}

Recently, the hazards of using herbicide have been accentuated by a large scale in agriculture to control the growth of broad-leaved weeds on rice, maize, wheat, and in post emergence applications. Among the most usually used herbicides compounds, 2,4-dichlorophenoxyacetic acid (2,4-D) is a common and important phenoxy herbicide which has been frequently detected in water (Garabrant and Philbert, 2002; Omidi et al., 2014). It belongs to the top10 hit-list of pesticides used all over the world since it has strong endocrine-disrupting activities (Wang et al., 2013). The World Health Organization (WHO) and United States Environmental Protection Agency (EPA) have regulated $70 \mu \mathrm{g} \mathrm{L}^{-1}$ as the maximum contaminant level

\footnotetext{
* Corresponding authors.

E-mail addresses: dzshen@sdnu.edu.cn (D. Shen), lxchen@yic.ac.cn (L. Chen).
}

(MCL) of 2,4-D in drinking water (Hamilton et al., 2003; Han et al., 2010). Considering its healthy and toxicological effects, the recognition and detection of 2,4-D at low concentrations is of great importance and a major challenge. Conventional routine analytical techniques typically include high performance liquid chromatography (HPLC) coupled with UV detector (HPLC-UV) (Behbahani et al., 2014; Biesaga et al., 2005) or mass spectrometry (MS) (HPLC-MS) (Beeson et al., 1999; Luo and Yu, 2014), and gas chromatography (GC) (Rezazadeh et al., 2014) and GC-mass spectrometry (GC-MS) (Catalina et al., 2000; Chouhan et al., 2006; Maloschik et al., 2010; Rodríguez et al., 2005). However, they often require extensive sample preparation and separation procedures, owing to the complexity of the sample matrices and the low content of 2,4-D. Also, they are still involved in some problems such as complicated instruments, high cost, time-consuming process, and particularly low selectivity. Therefore, molecularly imprinted polymers (MIPs) with desired selectivity, physical robustness, thermal stability, as well as low cost and easy preparation (Li et al., 
2011), are gaining popularity in sample pretreatment (Chen et al. 2011; Liu et al., 2014; Omidi et al., 2014; Yang et al., 2013; Zhong et al., 2014; Zhou et al., 2015) and sensors (Anirudhan and Alexander, 2014; Guan et al., 2011; Wang et al., 2013, 2015) for determination of trace 2,4-D in complex matrices.

On the other hand, fluorescent detection has attracted a great deal of interest for various chemically, environmentally, and biologically significant species such as 2,4-D (Vinayaka et al., 2009), owing to the capabilities of high sensitivity and convenience (Geng et al., 2010; Vinayaka et al., 2009; Yu et al., 2015; Zhang et al., 2015a; Zhu et al., 2014). It is considered to be an efficient way to develop MIP-based fluorescence sensors, which will combine the advantages of high selectivity of MIPs recognition and high sensitivity of fluorescence detection (Vinayaka et al., 2009; Wu et al. 2015; Zhang et al., 2015b). To date, most of the sensors are focused on fluorescence turn off, in which the fluorescence signal is diminished in the presence of the target analyte (Wu et al., 2015; Zhang et al., 2015b). Turn on fluorescence strategy is less commonly reported, but it is highly attractive because of the improved sensitivity by the enhancement of a fluorescence signal. However, either turn off or turn on fluorescence intensity as a sole responsive signal, the signal is easily interfered by various experimental factors, such as the change in fluorescence intensity and instrumental conditions. Excitingly, ratiometric measurements have the advantages to eliminate these environmental effects and give more precise measurement owing to their self-referencing capability by calculation of two emission intensity ratio instead of the absolute intensity of one peak (Liu et al., 2013; Wang et al., 2016; Zhang et al., 2014). In general, two individual materials with different fluorescence emission wavelengths can be used to build ratiometric sensors (Xu and Lu, 2015). As the two different emissions have different responses to analytes, one emission peak can be used as specific recognition signal for the target analyte, while the other one is constant as reference signal. Thus, the sensors can clearly change fluorescence colors during sensing, which can be observed by the naked eye (Lan et al., 2014).

Inspired by these studies, herein, we expect to construct a novel molecular imprinting-based turn on ratiometric fluorescence sensor for visual identification and detection of 2,4-D, combining the green nitrobenzoxadiazole (NBD) fluorophore and red quantum dots (QDs) embedded in silica nanoparticles as signal sources. Silica nanoparticles were used as the support materials, which is mainly due to two reasons. One reason is that they are optically transparent and inert, which will prevent the direct contact of red QDs with the external analytes, providing a reliable reference signal for the ratiometric detection. The other reason is that their surface can readily be modified by coupling reactions with alkoxysilane derivatives. By using the red QD-embedded silica nanoparticles as core, the imprinted shell was prepared on the surface of red QD@SiO ${ }_{2}$ by sol-gel method using 2,4-D as template, 3-aminopropyl triethoxysilane (APTES) as functional monomer and tetraethoxysilicane (TEOS) as cross-linker with NBD as a signal unit. The strategy for designing fluorescence sensor was based on the occurrence of photoinduced electron transfer process (PET) between NBD and the amine groups of APTES, since the amine group with nitrogen lone-pair electron can quench a nearby NBD fluorophore through an N-to-NBD electron transfer. When the amine groups bound with 2,4-D via hydrogen bond, a dramatic enhancement in the fluorescence intensity could be observed because PET process was inhibited, while the fluorescence intensity of red QDs maintained constant. As the concentration of 2,4-D increased, the two emission intensity ratios gradually varied and led to continuous changes of fluorescence color from orange red to green, which could be clearly observed by the naked eye. On the basis of the imprinting recognition and turn on ratiometric fluorescence with color change, the MIP-based fluorescence sensor could be practically applied for highly selective and sensitive determination of 2,4-D in water samples.

\section{Experimental}

\subsection{Reagents and materials}

4-Chloro-7-nitro-2,1,3-benzoxadiazole (NBD-Cl) and 3-aminopropyltriethoxysilane (APTES) were purchased from Sigma-Aldrich (Shanghai, China). Ammonium hydroxide (25\%), absolute ethanol, methanol, acetic acid (HAc), Tellurium powder, cadmium nitrate $\left(\mathrm{Cd}\left(\mathrm{NO}_{3}\right)_{2}\right)$, tetraethoxysilicane (TEOS) and phosphate buffered saline (PBS) were supplied by Sinopharm Chemical Reagent Co. Ltd. (Shanghai, China). 2,4-dichlorophenoxyacetic acid (2,4-D), phenoxyacetic acid (PA), 2,4-dichlorophenylacetic acid (DCPA) and 4-chloro-2-methylphenoxyacetic acid (MCPA) were purchased from J\&K Technology Ltd. (Beijng, China).

\subsection{Characterization}

Fluorescence measurements were taken on a Fluoromax-4 Spectrofluorometer (Horiba Scientific). The morphological evaluation was recorded by a transmission electron microscopy (TEM, JEM-1230, operating at $100 \mathrm{kV}$ ) and scanning electron microscope (SEM, Hitachi S-4800 FE-SEM, operating at $5 \mathrm{kV}$ ).

\subsection{Preparation of MIP-based fluorescence sensor}

Red emissive CdTe QDs were firstly synthesized in aqueous phase as described (Zhang et al., 2010). Then, the red QD-embedded silica nanoparticles (QD@SiO ${ }_{2}$ ) were synthesized by reverse microemulsion according to the report method (Wang et al., 2009). Meanwhile, the APTES-NBD conjugates were synthesized using our developed method (Wang et al., 2016). Afterwards, the ratiometric fluorescence MIPs sensor, marked as QD@SiO ${ }_{2} @ N B D @ M I P s$, was prepared by a facile sol-gel process. Typically, $1 \mathrm{~mL}$ APTES-NBD conjugates solution and $20 \mathrm{mg}$ of 2,4-D were added to $25 \mathrm{~mL}$ of ethanol containing QD@SiO ${ }_{2}$ nanoparticles. After stirred for $30 \mathrm{~min}, 100 \mu \mathrm{L}$ of $\mathrm{NH}_{3} \cdot \mathrm{H}_{2} \mathrm{O}$ and $50 \mu \mathrm{L}$ of TEOS were added and kept stirring overnight in the dark. Finally, the products were centrifuged and washed with methanol/HAc $(9: 1, v / v)$ to remove $2,4-\mathrm{D}$, and then washed to neutral

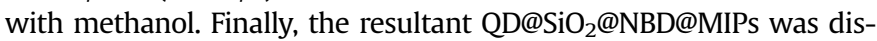
persed in $10 \mathrm{~mL}$ ethanol for further use. For comparison, $\mathrm{SiO}_{2} @ \mathrm{NBD} @ \mathrm{MIPs}$ were prepared in the same manner but without embedding red QDs. As a control, non-imprinted sensor, marked as QD@SiO ${ }_{2} @ N B D @ N I P s$, was synthesized under the identical conditions in the absence of the template 2,4-D.

\subsection{Fluorescence measurement}

All the fluorescence $(\mathrm{FL})$ intensities were measured under the same conditions: the excitation and emission slit widths were both $4 \mathrm{~nm}$ and the excitation wavelength was set at $420 \mathrm{~nm}$ with a recording emission range of 440-700 nm. QD@SiO ${ }_{2} @ N B D @ M I P s$, $\mathrm{SiO}_{2} @ N B D @ M I P s$ and QD@SiO ${ }_{2} @ N B D @ N I P s$ were added into the solutions containing known concentrations of 2,4-D, and the final concentration of MIPs or NIPs in ethanol was $0.26 \mathrm{mg} \mathrm{mL}^{-1}$.

\subsection{Analysis of water samples}

Water samples including lake water and tap water were used to demonstrate the practical application of the QD@SiO ${ }_{2} @ N B D @ M I P s$ for the detection of 2,4-D. Lake water sample was collected from San Yuan Lake, located in the coastal zone of Yantai City, and tap water sample was acquired in our laboratory after flowing for 
about $5 \mathrm{~min}$. The real water samples were first filtered with $0.45 \mu \mathrm{m}$ microfiltration membrane to remove any possible particulate suspension, and then they were diluted 100 -fold for spiking. The spiked water sample with known concentrations of 2,4-D were used to validate the accuracy and applicability of this QD@SiO ${ }_{2} @ N B D @ M I P s$ sensor.

\section{Results and discussion}

\subsection{Preparation and detection principle of QD@SiO ${ }_{2} @ N B D @ M I P s$}

Scheme 1 illustrates the preparation process and possible detection principle of QD@SiO ${ }_{2} @ N B D @ M I P s$ sensor for fluorescence turn on and ratiometric detection of 2,4-D. As can be seen, the red CdTe QDs were firstly synthesized in aqueous phase and $\mathrm{QD} @ \mathrm{SiO}_{2}$ composite nanoparticles were prepared by reverse microemulsion method at room temperature. The resultant $\mathrm{QD} @ S i O_{2}$ nanoparticles had a fluorescence maximum at about $655 \mathrm{~nm}$ and showed strong red fluorescence under a UV lamp. The red QDs were fully embedded in the $\mathrm{SiO}_{2}$ nanoparticles to prevent direct contact with external 2,4-D and provided a reliable reference signal for the ratiometric detection. Then the 2,4-D imprinted shell was formed on the surface of $\mathrm{QD} @ \mathrm{SiO}_{2}$ nanoparticles by one simple facile sol-gel polymerization step, using APTES as functional monomer, TEOS as cross-linker, and APTES-NBD conjugates as a signal recognition unit. When APTES and NBD were immobilized simultaneously on the surface of silica nanoparticles, a charge transfer could occur between the electron-rich amino group of APTES and the electron-deficient aromatic ring of NBD. Thus, the fluorescence of NBD would be partially quenched (fluorescence OFF) by the nitrogen atom of the free primary amine through a PET process. In the presence of 2,4-D, hydrogen bond would form between amino and carboxyl group, and the resultant positively charged amine $\left(\mathrm{NH}_{3}{ }^{+}\right)$could effectively decrease the electron-donating ability of nitrogen atoms and thereby switch off the PET pathway, so a dramatic enhancement of the fluorescence intensity (fluorescence $\mathrm{ON}$ ) at approximately $535 \mathrm{~nm}$ would be observed, as shown in Scheme 1.

As the concentration of 2,4-D increased, the green fluorescence of NBD was enhanced, whereas the red fluorescence of QDs was inert toward 2,4-D with almost unchanged intensity and hence could be used as stable internal reference (Scheme 1). As a result, the dual emission intensity ratios $\left(I_{535} / I_{655}\right)$ of the $\mathrm{QD} @ \mathrm{SiO}_{2} @ \mathrm{NBD} @ \mathrm{MIPs}$ sensor gradually increased. Thus, the ratiometric fluorescence

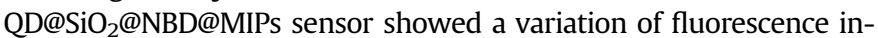
tensity ratios and a clear continuous color change from orange red to green with the addition of 2,4-D, which can be conveniently observed by the naked eyes under UV irradiation and thus facilitate the detection of 2,4-D. Therefore, the molecular-imprinting based

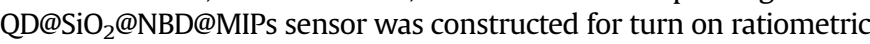
detection of 2,4-D.

\subsection{Morphological structure and optical properties of QD@SiO $@ @ N B D @ M I P s$}

The morphological structure and optical properties of the obtained QD@SiO $\mathrm{S}_{2} @$ NBD@MIPs were studied in detail. Fig. 1 shows the morphological structures of $\mathrm{QD} @ \mathrm{SiO}_{2}, \mathrm{QD} @ \mathrm{SiO}_{2} @ \mathrm{NBD} @ \mathrm{MIPs}$ and QD@SiO ${ }_{2} @ N B D @ N I P s$ by TEM and SEM. As seen, the red $\mathrm{QD} @ \mathrm{SiO}_{2}$ nanoparticles had good dispersion and smooth surfaces with the average diameter about $50-70 \mathrm{~nm}$ (Fig. 1A and a). As for the MIPs and NIPs, similar morphology could be obviously observed on the red QD@SiO ${ }_{2}$ nanoparticles (Fig. 1B and C). Meanwhile, the rough surfaces of MIPs and NIPs (Fig. 1b and c) also indicated the existence of shell. And the shell thicknesses were about $5-10 \mathrm{~nm}$ calculated from the TEM photo, verifying the successful introduction of shell on the surface of silica nanoparticles. The ultrathin imprinting shell layer would facilitate higher sensitivity and shorter analysis time of the sensor.

The fluorescence spectra of NBD, CdTe QDs, and the sensor were recorded in Fig. 2A. As seen, the green fluorescence NBD (insert a) showed fluorescence maximum at $535 \mathrm{~nm}$ (curve a), while the red fluorescence QDs (insert c) showed fluorescence maximum at $655 \mathrm{~nm}$ (curve c). When the two different colors of fluorescence materials were incorporated together, the ratiometric fluorescence sensor possessed dual emission peaks at 535 and $655 \mathrm{~nm}$ under a single wavelength excitation of $420 \mathrm{~nm}$ (curve b), and displayed orange red fluorescence (insert b). These results clearly suggested that the ratiometric fluorescence sensor was successfully prepared.

The effect of $\mathrm{pH}$ was evaluated in terms of the enhancing efficiency, defined as $\left(F-F_{0}\right) / F_{0}$ (Xu and Lu, 2015). As seen from Fig. $\mathrm{S} 1$, the enhancing efficiency increased below $\mathrm{pH} 7.5$ and decreased as $\mathrm{pH}$ values increased. These results showed that $\mathrm{pH} 7.5$ was the optimum $\mathrm{pH}$ value for detection of 2,4-D. And then $\mathrm{pH}$ was fixed at 7.5. Further experiments were carried out at $\mathrm{pH} 7.5$.

The fluorescence stabilities of QD@SiO $\mathrm{SNBD}_{2} @ \mathrm{MIPs}$ were estimated during the storage by repeatedly detecting the fluorescence intensity every day at room temperature. It was found that the fluorescence intensity of the QD@@SiO ${ }_{2} @ N B D @ M I P s$ retained almost no change when the MIPs were stored for 10 days (data not shown), which implied that the resultant MIPs displayed good physical stability due to the excellent protection of MIP coatings.

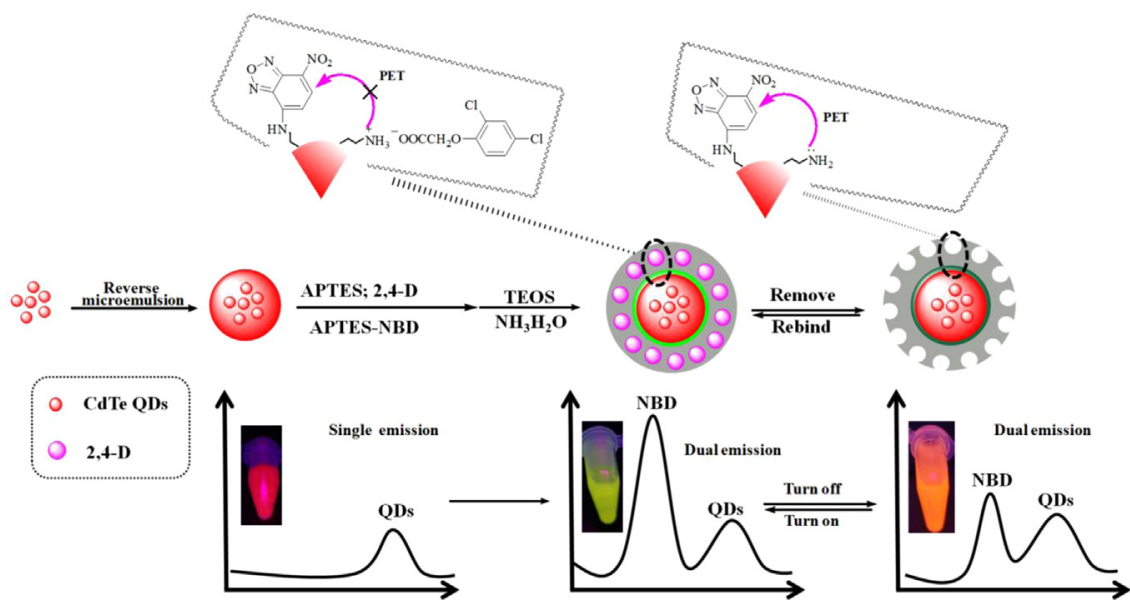

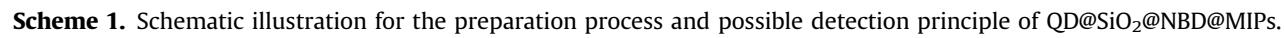



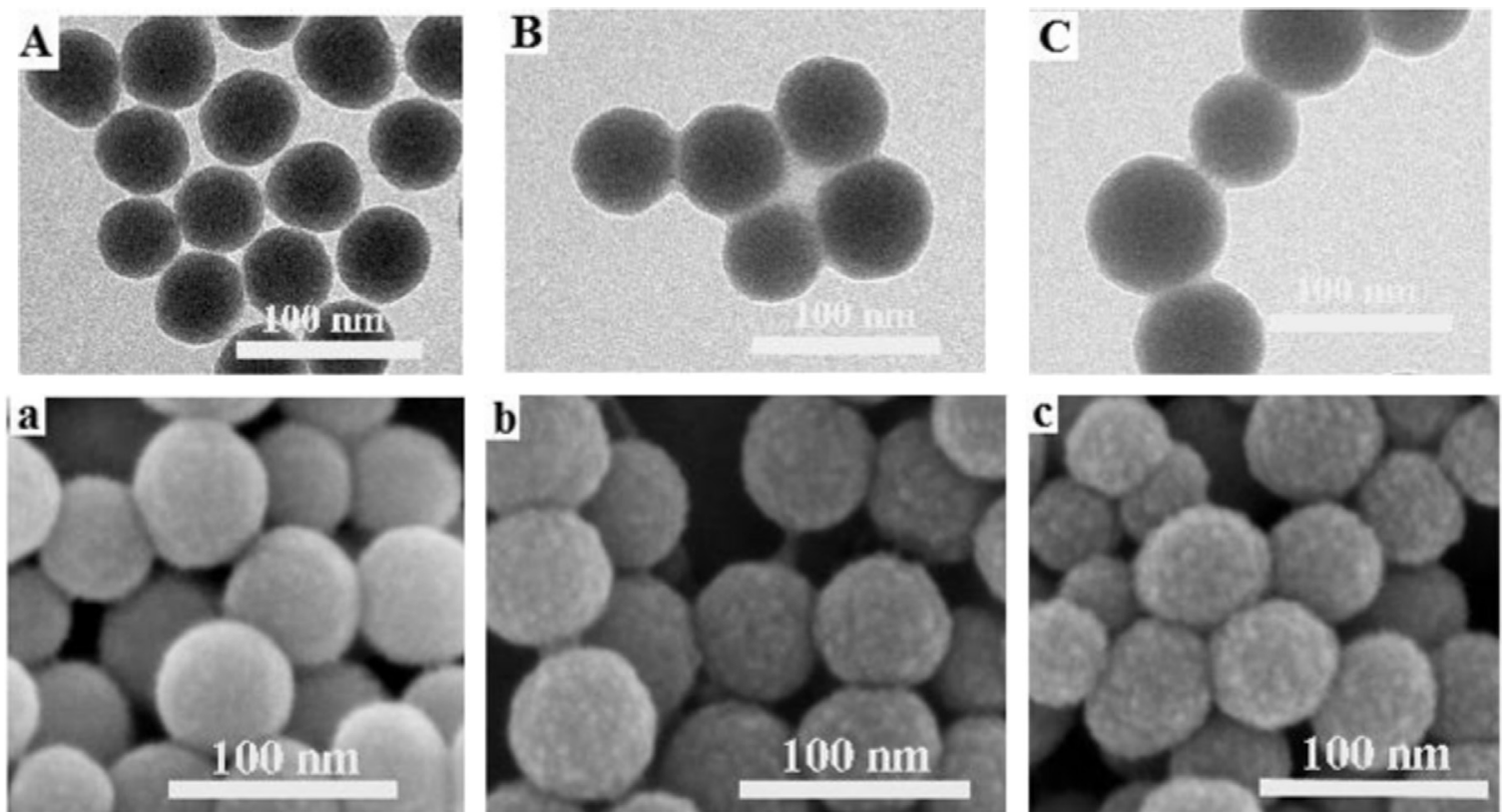

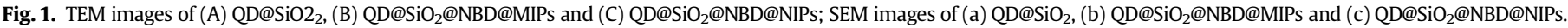

A

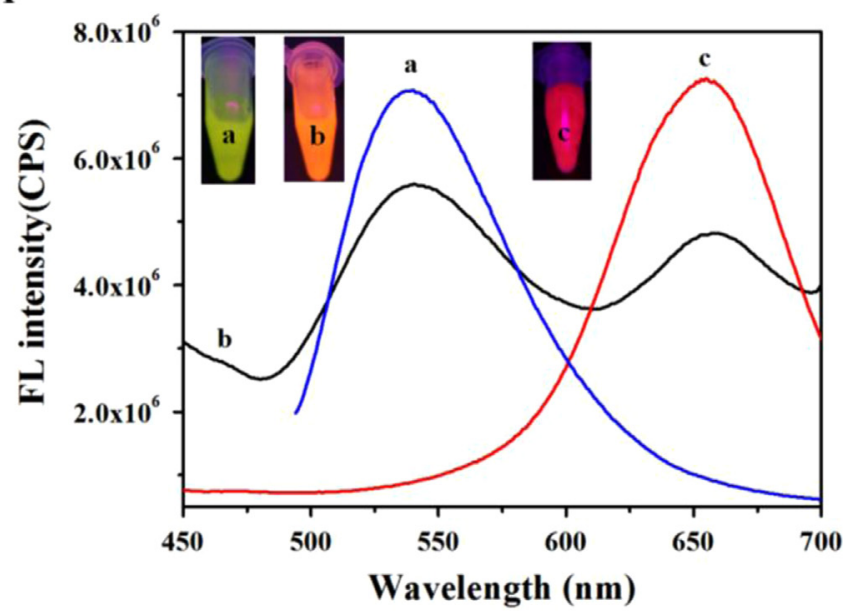

B

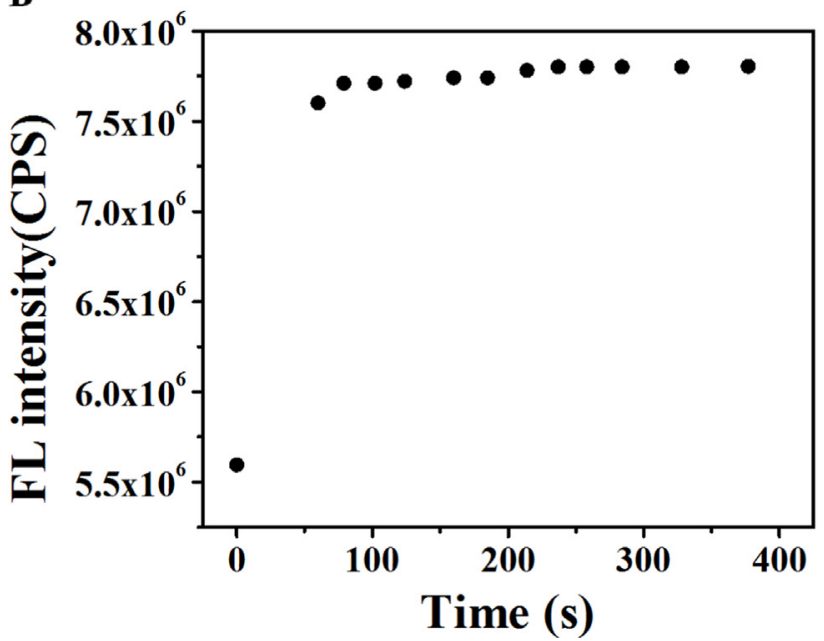

Fig. 2. (A) Fluorescence emission spectra of (a) green emissive NBD, (b) $\mathrm{QD} @ \mathrm{SiO}_{2} @ \mathrm{NBD} @ \mathrm{MIPs}$, and (c) red emissive $\mathrm{QD} @ \mathrm{SiO}_{2}$. The inset photos show the corresponding fluorescence colors under a $365 \mathrm{~nm}$ UV lamp, respectively. (B) Fluorescence response time of 2,4-D imprinted QD@SiO ${ }_{2} @ N B D @ M I P s$ for 2,4-D. The fluorescence intensity was recorded at the wavelength of $535 \mathrm{~nm}$.
The response time of the ratiometric fluorescence sensor was also investigated to assess the accessibility to binding sites. As seen from Fig. 2B, the fluorescence intensity at $535 \mathrm{~nm}$ increased fast within 5 min when the concentration of 2,4-D was $50 \mu \mathrm{M}$, after which the curve became flat. The thin imprinting layer with

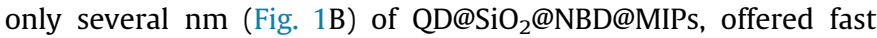
mass transfer and high site accessibility toward the template, resulting in short response time of 2,4-D. Therefore, 5 min was chosen as the response time for further experiments.

\subsection{Sensitivity and selectivity of the sensor}

The ability of the QD@SiO ${ }_{2} @ N B D @ M I P s$ sensor for quantitative analysis of 2,4-D was further evaluated. Under the optimized para-

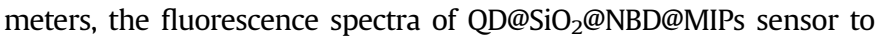
different concentrations of 2,4-D were recorded for sensitivity investigation. As seen from Fig. 3A, the fluorescence emission intensity of the green NBD at $535 \mathrm{~nm}$ gradually increased since the PET process was inhibited, whereas the one at $655 \mathrm{~nm}$ of the red QDs showed only a little change. As a result, the emission intensity ratio, $I_{535} / I_{655}$, increased gradually with the concentration increase of $2,4-\mathrm{D}$, along with a continuous variation of the fluorescence colors from orange red to green under a $365 \mathrm{~nm}$ UV lamp, as shown in the insets of Fig. 3A. This turn on and ratiometric fluorescence enhancement could be easily observed with a distinguishable color change, which was greatly favorable for visual detection of 2,4-D by the naked eye. Consequently, an excellent linearity was presented within a wide range of $0.4-100 \mu \mathrm{M}$. Based on $3 \sigma / s$, in which $\sigma$ means the standard deviation of the blank measurements, and $s$ means the slope of calibration curve, the limit of detection (LOD) was attained as low as $0.14 \mu \mathrm{M}$. The value is lower than the MCL for 2,4-D in drinking water, i.e., $0.32 \mu \mathrm{M}\left(70 \mu \mathrm{g} \mathrm{L}^{-1}\right)$ regulated by the WHO and EPA (Hamilton et al., 2003; Han et al., 2010).

The advantages of the ratiometric fluorescence sensor for visual detection of 2,4-D can be clearly noticed by comparison with the single fluorescence enhancing experiments (Fig. 3B), in which red QDs were omitted in preparation of the senor. As can be seen from the inset of Fig. 3B, the color changes of the pure NBD were hard to see by the naked eye with the addition of 2,4-D, and meanwhile the linear range was narrow. The comparison clearly demonstrated that 
A

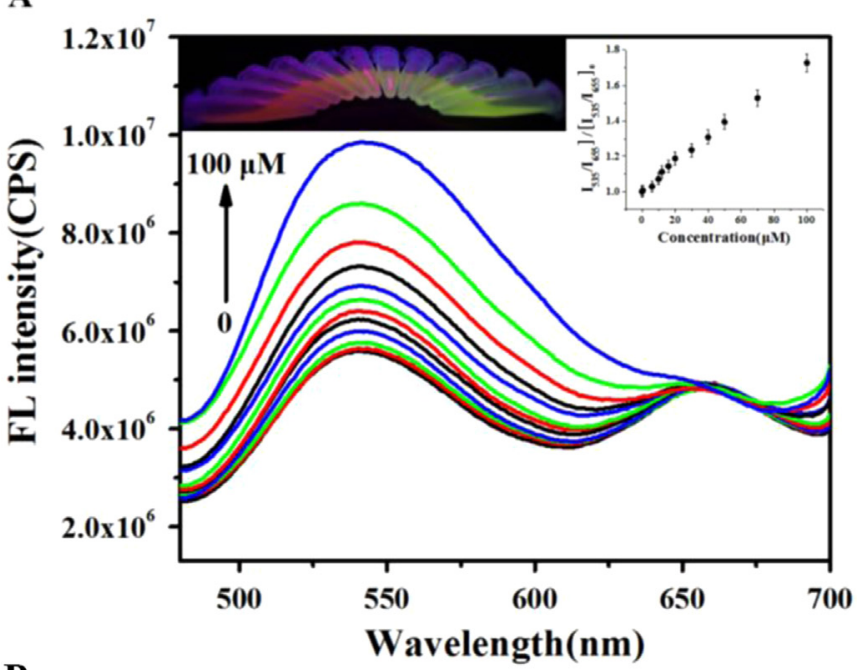

B

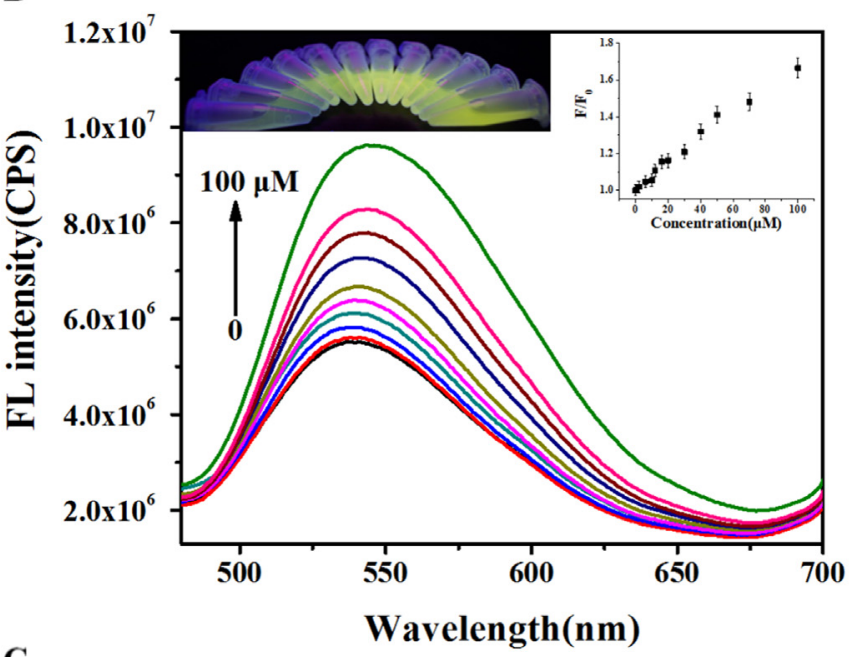

C

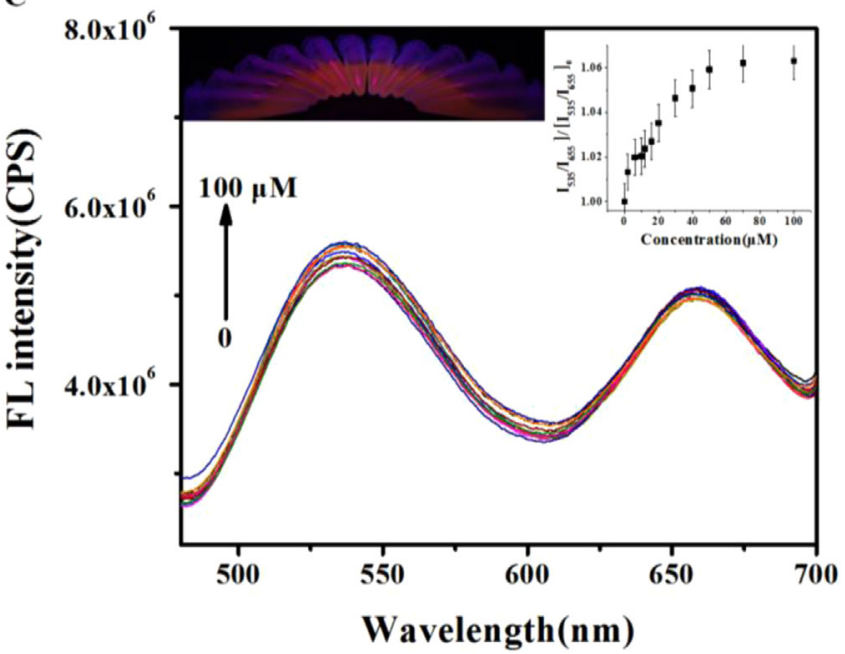

Fig. 3. Fluorescence spectra, corresponding fluorescence colors (inset, from left to right), and relationships between fluorescence ratios and the concentrations (inset) of 2,4-D from 0 to $100 \mu \mathrm{M}$ for (A) QD@SiO ${ }_{2} @ N B D @ M I P s,(B) S_{2} O_{2} @ N B D @ M I P s$, and (C) QD@SiO $@$ NBD@NIPs. Experimental conditions: concentration of MIPs or NIPs in ethanol was $0.26 \mathrm{mg} \mathrm{mL}^{-1}$; excited light, $420 \mathrm{~nm}$; silt widths of excitation and emission, $4 \mathrm{~nm}$.

the ratiometric fluorescence sensor was more sensitive and reliable for visual detection than a single fluorescence enhancing method.

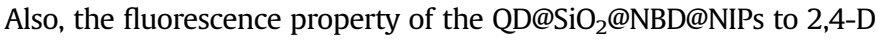

was examined. As seen from Fig. 3C, the fluorescence intensity of NIPs could also be enhanced by 2,4-D. However, the sensitivity was much lower and the linear range was narrower, and meanwhile the fluorescence color change was not obvious. As observed from Fig. 3A and C, MIPs and NIPs offered different linear relationships, and the increase of fluorescence intensity of MIPs was much larger than that of the NIPs at the same 2,4-D concentration. So, a high imprinting factor could be obtained of 4.97. This phenomenon can be explained that no recognition sites existed in the NIPs, so 2,4-D cannot enter into the inner of the NIPs, and the fluorescence intensity of the NBD can only be enhanced by non-specific adsorption of 2,4-D, resulting in slight enhancement of the fluorescence intensity. Therefore, all the results indicated the MIPs based dual emitting ratiometric fluorescence sensor could highly sensitively and accurately recognize and detect 2,4-D, and thereby implied its feasibility for fluorescently visually monitoring phenoxy herbicide pollution.

On the other hand, the selectivity of the MIPs ratiometric fluorescence sensor was also further evaluated by recording the fluorescence emission ratios $\left(I_{535} / I_{655}\right)$ of the sensor in the presence of 2,4-D and its analogs, including PA, DCPA and MCPA. As shown in Fig. 4, the fluorescence intensity at $535 \mathrm{~nm}$ of the MIPs sensor was enhanced to be about $71.7 \%$ by $2,4-\mathrm{D}$, while only less than $19.5 \%$ by the analogs. Meanwhile, obviously, green fluorescence color was observed in the presence of 2,4-D on the original background, however, no fluorescence color changes happened after adding the analogs, which almost did not turn on the fluorescence emission at $535 \mathrm{~nm}$ of the sensor, as shown in the inset of Fig. 4. Moreover, it was found that the NIPs sensor showed similar low fluorescence emission ratios for 2,4-D and its analogs. Hence, the MIPs sensor had high selectivity toward the template molecule 2,4-D for fluorescent visual detection.

\subsection{Practical application of the turn on Ratiometric sensor to real water samples}

To further assess the applicability of the turn on ratiometric fluorescence sensor, the detection of 2,4-D in real water samples including lake water and tap water was carefully demonstrated by recovery tests. The averaged recovery was obtained with relative standard deviation (RSD) based on three triplicate measurements for each concentration. Table 1 lists the results for the samples with and without 2,4-D spiked. Clearly, the recoveries of 2,4-D were

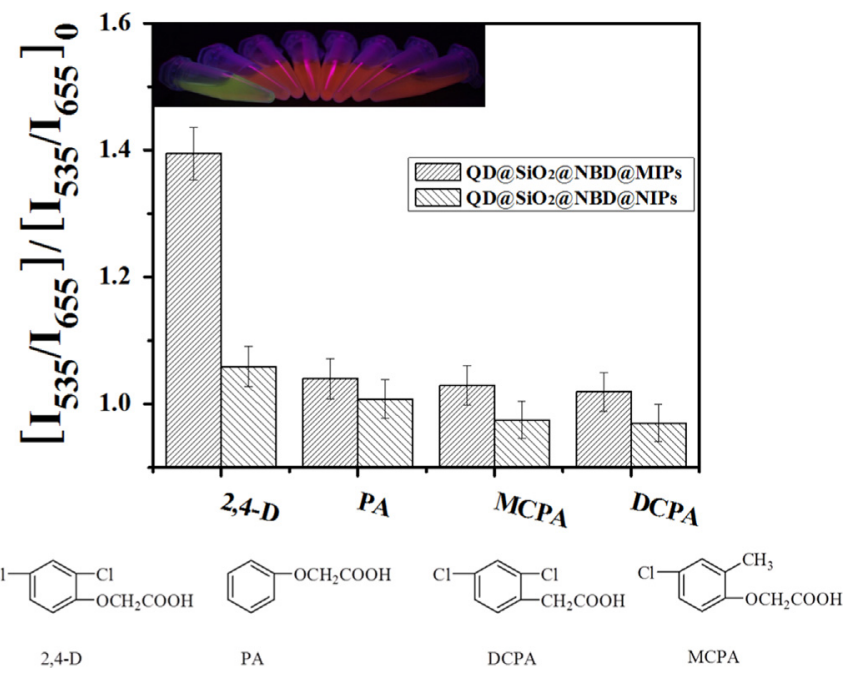

Fig. 4. Selectivity of QD@SiO $\mathrm{S}_{2} @ N B D @ M I P s$ and $\mathrm{QD} @ \mathrm{SiO}_{2} @ N B D @ N I P s$ for 2,4-D over its analogs including PA, MCPA and DCPA at concentration of $50 \mu \mathrm{M}$, and the inset shows the corresponding fluorescence color change of the tested solutions from left to right. Concentration of MIPs or NIPs was in ethanol $0.26 \mathrm{mg} \mathrm{mL}^{-1}$; excited light, $420 \mathrm{~nm}$; silt widths of excitation and emission, $4 \mathrm{~nm}$. 
Table 1

Spiked recoveries and RSDs (\%; $n=3)$ for the determination of 2,4-D in lake water

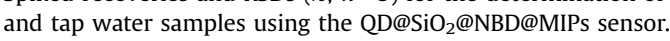

\begin{tabular}{lccl}
\hline Sample & Added $(\mu \mathrm{M})$ & Found $(\mu \mathrm{M})$ & Recovery \pm RSD $(\%)$ \\
\hline Lake water & 0 & 0 & - \\
& 0.50 & 0.475 & $95.0 \pm 3.5$ \\
& 4.00 & 4.14 & $103.5 \pm 3.6$ \\
Tap water & 10.00 & 11.01 & $110.1 \pm 4.5$ \\
& 0 & 0 & - \\
& 0.50 & 0.481 & $96.2 \pm 3.9$ \\
& 4.00 & 3.92 & $98.0 \pm 4.1$ \\
& 10.00 & 10.23 & $102.3 \pm 3.8$ \\
\hline
\end{tabular}

statistically close to that of the spiked values, suggesting that there are no serious positive or negative interferences in real water samples and satisfactory recoveries were attained of $95.0-110.1 \%$ with RSDs of 3.5-4.5\% for the spiked lake water samples and 96.2-102.3\% with RSDs of $3.8-4.1 \%$ for the spiked tap water samples, respectively. The results indicated that the ratiometric fluorescence sensor was suitable for 2,4-D determination in real water samples, possessing great potential for practical applications.

\subsection{Method performance comparison}

The performance of the developed ratiometric fluorescence method for detection of 2,4-D was compared with some reported MIPs-based approaches such as HPLC and chemiluminescence, as listed in Table S1. It can be seen that the reported MIPs coupled with HPLC methods often involve complicated sample treatment process and require long analysis time (Liu et al., 2014; Yang et al., 2013; Zhong et al., 2014; Zhou et al., 2015). Yu's group (Wang et al., 2013) has reported a kind of paper-based MIPs for chemiluminescence detection of 2,4-D at the concentration of femtomolar level and the response time is only $4 \mathrm{~min}$. However, the synthesis procedure for the MIPs is timeconsuming and hard to control. Excitingly, our developed turn on ra-

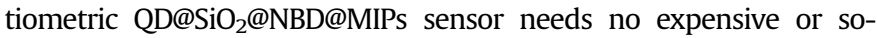
phisticated instrumentation, or complicated sample preparation, and the obtained LOD is comparable to or lower than that of MIP-SPEHPLC methods. Furthermore, our constructed ratiometric 2,4-D detection system provides convenience for the naked eye detection without the need for sophisticated equipments except for a small UV lamp, and the it is timesaving within 5 min to complete the detection. As a result, the present analytical method in our study has remarkable advantages such as simplicity, rapidity and visualization, high selectivity and sensitivity, and good reliability and practicability.

\section{Conclusions}

In conclusion, a simple 2,4-D imprinted QD@SiO $2 @ N B D @ M I P s$ dual-emission fluorescence sensor was constructed through a facile sol-gel polymerization, and successfully applied to water samples for detection of 2,4-D based on PET. With the presence of 2,4-D, the green emission (NBD) of the sensor was enhanced owing to the PET process inhibited, while the red emission (QDs) was almost unchanged as stable internal reference, for visual detection of 2,4-D via turn on and ratiometric fluorescence. By virtue of the synergy of highly selective MIPs and highly sensitive fluorescence, the sensor demonstrated high selectivity and sensitivity for recognition and determination of 2,4-D, along with visual signal output observed by the naked eye. Also, rapid response, high accuracy and stability, and feasible practicability were all attained. The sensor can be used as an ideal alternative analytical tool for 2,4-D, which will favorably contribute to water pollution monitoring and abatement.
On the other hand, environmentally friendly fluorescence signal nanomaterials are particularly promising and should be strongly explored. The recently emerging silica QDs and carbon dots, as new types of safe and cheap QDs labels, have inspiring prospects in environmental and even clinical applications. Some are readily synthesized or commercially available, and consequently our present sensing strategy can offer exciting perspectives for fabrication of visual fluorescence sensors towards various organic and biological molecules. Moreover, eco-benign and ingenious preparative technologies should be continuously exploited in order to smartly combining and fully utilizing imprinting recognition and fluorescent detection for significantly improving the sensing performances avoiding potential risks. Furthermore, the fluorescence turn on and/ or ratiometric types are also highly desirable and need more explorations, as well as possible sensing principles. Such proposed related research explorations will greatly promote the development of intelligent fluorescent sensors, and broaden the research connotations of concerned-target imprinting.

\section{Acknowledgments}

This work was financially supported by the National Natural Science Foundation of China (21575080, 21275091, 21275158, 21477160), the Research Fund for the Doctoral Program of Higher Education of China (20113704110003), and the Scientific Research Foundation for the Returned Overseas Chinese Scholars, State Education Ministry.

\section{Appendix A. Supporting information}

Supplementary data associated with this article can be found in the online version at http://dx.doi.org/10.1016/j.bios.2016.03.031.

\section{References}

Anirudhan, T.S., Alexander, S., 2014. Appl. Surf. Sci. 303, 180-186.

Beeson, M.D., Driskell, W.J., Barr, D.B., 1999. Anal. Chem. 71, 3526-3530.

Behbahani, M., Najafi, F., Bagheri, S., Bojdi, M.K., Hassanlou, P.G., Bagheri, A., 2014. Environ. Monit. Assess. 186, 2609-2618.

Biesaga, M., Jankowska, A., Pyrzyńska, K., 2005. Microchim. Acta 150, 317-322.

Catalina, M.I., Dallüge, J., Vreuls, R.J., Brinkman, U.A.T., 2000. J. Chromatogr. A 877 , $153-166$.

Chen, L.X., Xu, S.F., Li, J.H., 2011. Chem. Soc. Rev. 40, 2922-2942.

Chouhan, R.S., Babu, K.V., Kumar, M.A., Neeta, N.S., Thakur, M.S., Rani, B.E.A., Pasha, A. Karanth, N.G.K., Karanth, N.G., 2006. Biosens. Bioelectron. 21, 1264-1271. Garabrant, D.H., Philbert, M.A., 2002. CRC Crit. Rev. Toxicol. 32, 233-257.

Geng, J.L., Liu, P., Liu, B.H., Guan, G.J., Zhang, Z.P., Han, M.Y., 2010. Chem. Eur. J. 16, 3720-3727.

Guan, G.J., Wang, S.S., Zhou, H.B., Zhang, K., Liu, R.Y., Mei, Q.S., Wang, S.H., Zhang, Z P., 2011. Anal. Chim. Acta 702, 239-246.

Hamilton, D., Ambrus, A., Dieterle, R., Felsot, A., Harris, C., Holland, P., Katayama, A., Kurihara, N., Linders, J., Unsworth, J., Wong, S., 2003. Pure Appl. Chem. 75, $1123-1155$.

Han, D.M., Jia, W.P., Liang, H.D., 2010. J. Environ. Sci. 22, 237-241.

Lan, M.H., Zhang, J.F., Chui, Y.S., Wang, P.F., Chen, X.F., Lee, C.S., Kwong, H.L., Zhang, W.J. 2014. ACS Appl. Mater. Interfaces 6, 21270-21278.

Li, J.H., Zhang, Z., Xu, S.F., Chen, L.X., Zhou, N., Xiong, H., Peng, H.L., 2011. J. Mater. Chem. 21, 19267-19274.

Liu, B.Y., Zeng, F., Wu, S.Z., Wang, J.S., Tang, F.C., 2013. Microchim. Acta 180, $845-853$.

Liu, Y.L., He, Y.H., Jin, Y.L., Huang, Y.Y., Liu, G.Q., Zhao, R., 2014. J. Chromatogr. A 1323, $11-17$.

Luo, C.Y., Yu, H.J., 2014. Chin. J. Health Lab Technol. 24, 3059-3061.

Maloschik, E., Mortl, M., Szekacs, A., 2010. Anal. Bioanal. Chem. 397, 537-548.

Omidi, F., Behbahani, M., Abandansari, H.S., Sedighi, A., Shahtaheri, S.J., 2014. J. Environ. Health Sci. Eng. 12, 137-147.

Rezazadeh, M., Yamini, Y., Seidi, S., Tahmasebi, E., Rezaei, F., 2014. J. Agric. Food Chem. 62, 3134-3142.

Rodríguez, I., Rubí, E., González, R., Quintana, J.B., Cela, R., 2005. Anal. Chim. Acta 537, 259-266.

Vinayaka, A.C., Basheer, S., Thakur, M.S., 2009. Biosens. Bioelectron. 24, 1615-1620. 
Wang, C., Ma, Q., Dou, W.C., Kanwal, S., Wang, G.N., Yuan, P.F., Su, X.G., 2009. Talanta 77, 1358-1364.

Wang, S.M., Ge, L., Li, L., Yan, M., Ge, S.G., Yu, J.H., 2013. Biosens. Bioelectron. 50, 262-268.

Wang, X.Q., Liu, X.Y., Wang, X.G., 2015. Sens. Actuators B Chem. 220, 873-879.

Wang, X.Y., Yu, J.L., Kang, Q., Shen, D.Z., Li, J.H., Chen, L.X., 2016. Biosens. Bioelectron. 77, 624-630.

Wu, X.Q., Zhang, Z., Li, J.H., You, H.Y., Li, Y.B., Chen, L.X., 2015. Sens. Actuators B Chem. 211, 507-514.

Xu, S.F., Lu, H.Z., 2015. Biosens. Bioelectron. 73, 160-166.

Yang, W.J., Jiao, F.P., Zhou, L., Chen, X.Q., Jiang, X.Y., 2013. Appl. Surf. Sci. 284, 692-699.

Yu, F.B., Gao, M., Li, M., Chen, L.X., 2015. Biomaterials 63, 93-101.

Zhang, K., Mei, Q.S., Guan, G.F., Liu, B.H., Wang, S.H., Zhang, Z.P., 2010. Anal. Chem. 82, 9579-9586.
Zhang, K., Yu, T., Liu, F., Sun, M.T., Yu, H., Liu, B.H., Zhang, Z.P., Jiang, H., Wang, S.H. 2014. Anal. Chem. 86, 11727-11733.

Zhang, Z., Li, J.H., Fu, L.W., Liu, D.Y., Chen, L.X., 2015a. J. Mater. Chem. A 3 , 7437-7444.

Zhang, Z., Li, J.H., Wang, X.Y., Shen, D.Z., Chen, L.X., 2015b. ACS Appl. Mater. Interfaces 17, 9118-9127.

Zhong, S.A., Zhou, C.Y., Zhang, X.N., Zhou, H., Li, H., Zhu, X.L., Wang, Y., 2014. J. Hazard. Mater. 276, 58-65.

Zhou, C.Y., Li, H., Zhou, H., Wang, H., Yang, P.J., Zhong, S.A., 2015. J. Sep. Sci. 38, 1365-1371.

Zhu, H.J., Yu, T., Xu, H.D., Zhang, K., Jiang, H., Zhang, Z.P., Wang, Z.Y., Wang, S.H. 2014. ACS Appl. Mater. Interfaces 6, 21461-21467. 